Are PEI-coated SWCNTs conjugated with hepatitis A virus? A chemical study with SEM, Zpotential, EDXD and RT-PCR

This article has been downloaded from IOPscience. Please scroll down to see the full text article.

2010 Biomed. Mater. 5035001

(http://iopscience.iop.org/1748-605X/5/3/035001)

View the table of contents for this issue, or go to the journal homepage for more

Download details:

IP Address: 160.80.2.8

The article was downloaded on 23/04/2010 at 10:35

Please note that terms and conditions apply. 


\title{
Are PEI-coated SWCNTs conjugated with hepatitis A virus? A chemical study with SEM, Z-potential, EDXD and RT-PCR
}

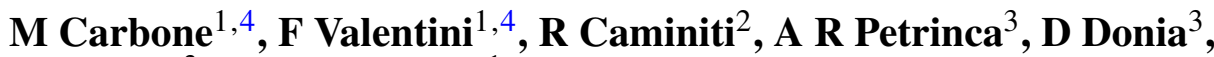 \\ M Divizia $^{3}$ and G Palleschi ${ }^{1}$ \\ ${ }^{1}$ Dip. Scienze e Tecnologie Chimiche, Università Tor Vergata, via della Ricerca Scientifica 1, \\ 00133 Roma, Italy \\ ${ }^{2}$ Department of Chemistry, University of "La Sapienza", P.le A. Moro 5, 00185, Roma, \\ Italy \\ ${ }^{3}$ Department of Public Health, Hygiene chair, University of Tor Vergata, Via Montpellier, n. 1, \\ 00133 Roma, Italy \\ E-mail: marilena.carbone@roma2.infn.it and federica.valentini@uniroma2.it
}

Received 17 September 2009

Accepted for publication 12 March 2010

Published 19 April 2010

Online at stacks.iop.org/BMM/5/035001

\begin{abstract}
The conjugation between nanotubes, coated with different doses of polyethylene imine (PEI) and hepatitis A virus (HAV) was investigated by scanning electron microscopy, Z-potential, thermogravimetric and differential thermal analysis, transmission electron microscopy, energy dispersive $\mathrm{x}$-ray diffraction (EDXD) and reverse transcript polymerase chain reaction

(RT-PCR). For the first time, to our knowledge, evidence is obtained that conjugation between the nanotubes and the HAV occurs and that it has an (at least a partial) electrostatic character. Since all components of the conjugated systems, nanotubes, coating material and virus are characterized by different peak shapes in the selected $q$ range, it was possible to infer that conjugation occurred. RT-PCR measurements confirmed that the conjugation of the coated nanotubes and HAV occurred and the result was stable. This opens up the prospect of probing the coated nanotubes as intra-cellular carriers in transfection processes of the virus. Further biological applications will concern a possible vaccine especially for non-replicative viruses.
\end{abstract}

(Some figures in this article are in colour only in the electronic version)

\section{Introduction}

Single-walled carbon nanotubes (SWCNTs) are versatile hydrophobic materials, which may be employed in several types of device including sensors, biosensors, electronic and optoelectronic devices [1] and field emission displays [2].

One of the main drawbacks in an efficient applicative use is related to their tendency to form aggregates, which causes poor solubility in all common solvents [3]. This initiated a large research stream of dispersion studies [4].

A way of circumventing the problem is coating of the nantotubes with polymers which yield composite materials,

\footnotetext{
4 Author to whom any correspondence should be addressed.
}

with properties modulated by the chosen polymer. Surface charge, degree of aggregation, solubility and branching of the coated material depend on the specific nanotube functionalization and coating.

The variety of functionalized, as well as coated SWCNTs, recently widened their application to the biological field [5], in particular as vaccine and drug delivery systems [6] or protein carriers [7]. Promising prospects, though, are related to virus transfection [8-11].

In such a framework, the choice of the coating material is fundamental. In a transfection process the virus has to be conjugated to the composite material, transferred through a membrane and released inside a cell: this all requires 
the possibility of modulating the carrier-virus interaction in different environments, through the proper polymer. The carrier-modulated transfection process can be particularly important for those viruses, which cannot multiply in cell lines because the specific receptors are absent on the cellular membrane. This is for instance the case of hepatitis A virus (HAV), which is able to multiply only on the FrHK cell line where it usually induces a characteristic cythopatic effect after 7-9 days post-infection. In this paper we present a study on the interaction between coated SWCNTs and HAV.

The aim is to analyse the possibility of using them as a vehicle to introduce the virus inside the non-permissive cells. In particular, the final goals are to produce a new progenia of virus to better understand the molecular biology of the virus; to prepare a possible vaccine specially for nonreplicative viruses; to produce more specific and sensible kits of diagnostic tools for environmental virology. Such a study poses a few questions on the type of coating polymer, and the ratio between nanotubes and polymer, that strongly influence the solubility and biocompatibility of the resulting nanocarriers. For this purpose, two functionalization procedures of the SWCNTs are widely used. The first one consists of the generation of carboxylic acid groups by oxidation with strong acids. This, usually, results in an overall shortening of the nanotubes (figure $1(a)$ ). The added oxygenated groups are, then, responsible for the carbon nanotube solubility in an aqueous polar medium [12] where homogeneous and stable dispersions are generated. A second functionalization procedure consists of coating the SWCNTs with polyelectrolytes that make them soluble in water $[13,14]$ or in a physiological medium [15] (figure 1(b)). The latter is a key prerequisite in considering the SWCNTs biocompatible for in vitro as well as in vivo experiments.

We opted for the polyelectrolyte polyethylene imine (PEI) which can be covalently conjugated to pre-functionalized carboxylated nanotubes [16]. This type of coating leaves a few ammonium heads free for further interaction and imprints the coated material with an overall positive charge. This, in turn, may provide an electrostatic interaction with, usually, negatively charged viruses such as the HAV (figure 1(c)).

The novelty of this paper concerns the conjugation of the HAV on the SWCNTs:PEI nanostructured platform, using different SWCTNs:PEI wt ratios of 1:2.5, 1:1, 1:0.5 and 1:0.2, respectively. As a characterization technique we choose energy dispersive $\mathrm{x}$-ray diffraction, since, in the proper ranges of reciprocal space, it offers immediate monitoring of the conjugation process. Furthermore, we used scanning electron microscopy, Z-potential and reverse-transcript polymerase chain reactions as control techniques.

For the first time, through these analytical tools, we have proven that the conjugation between the PEI-coated SWCNTs and the HAVs really occurs, and it resulted in a stable electrostatic interaction (as confirmed by the RT-PCR experiments).

\section{Materials and methods}

\subsection{Nanotube functionalization and conjugation procedure}

SWCNTs-COOH were purchased from CheapTubes (USA) and the $\mathrm{COOH}-\mathrm{SWNT}$ contain $2.7 \% \mathrm{COOH}$ groups (www.cheap tubes.com). SWCNTs-COOH were coated with PEI, following the EDC (1-ethyl-3-(3-dimethylaminopropyl) carbodiimide hydrochloride) chemical activation of the $\mathrm{COOH}$ groups on the SWCNT's walls, yielding a SWCNT/PEI composite (i.e.; 1:2.5; 1:1; 1:0.5 and 1:0.2\% w/w) [17]. Subsequently, the composite was dispersed in the phosphate buffer saline (PBS)/EtOH solution (at the concentration of $1 \mathrm{mg} \mathrm{ml}^{-1}$ ) using an ultrasonic bath for $30 \mathrm{~min}$, at room temparature. Then, $10^{6} \mathrm{HAV}$ virus particles were added to this homogeneous and stable dispersion, leaving HAV conjugates to the $\mathrm{COOH}-\mathrm{SWCNT} / \mathrm{PEI}$ composite at room temparature for $30 \mathrm{~min}$.

The mixture was gently washed three times with DMEM $1 \%$ FCS by centrifugation with a bench Eppendhorf centrifuge at $12000 \mathrm{rpm}$ for $5 \mathrm{~min}$ at room temperature. The presence of HAV was checked on the three washing buffers and SWCNT as reported.

\subsection{Thermogravimetric analysis and differential thermal analysis}

Thermogravimetric analysis (TGA)/differental thermal analysis (DTA) was performed by scanning from 20 to $870{ }^{\circ} \mathrm{C}$ under nitrogen at a heating rate of $20{ }^{\circ} \mathrm{C} \mathrm{min}-1$ by using a TGA: NETZSCH STA 409.

\subsection{Scanning electron microscope-energy dispersive $x$-ray detection}

Scanning electron microscope (SEM) images of all SWCNTs samples were taken using a FE-SEM (FE-SEM, LEO, model Supra 35) and EDXS (EDS, Oxford Instruments Ltd, model Inca 300). The samples were prepared by dropping $5 \mu \mathrm{L}$ of ethanol suspensions containing $1 \mathrm{mg} \mathrm{mL}^{-1}$ of SWCNTs-COOH, SWCNT/PEI composite, HAV virus and C-HAV (conjugated-HAV), on a fresh surface of silicon wafer, respectively; the sample was then allowed to dry at room temperature. The microscope was also equipped with an energy dispersive $\mathrm{x}$-ray detector for local quantitative elemental analysis of the samples.

\subsection{Transmission electron microscopy}

The SWNT-PEI 0.2 conjugated with the HAV virus and the virus, used here as control, were characterized by HRTEM using a TEM Philips (CM120, having LaB6 as filament) $100 \mathrm{kV}$ high-resolution scanning transmission electron microscope. For the TEM investigation, SWNT-PEI 0.2-HAV nanocomposite was previously dispersed in ethanol (1 mg/5 mL) using an ultrasonic bath for $1 \mathrm{~h}$, at room temperature. Following the same procedure, a HAV solution in ethanol has been prepared, and used here, as control. A perforated carbon Formvar film supported by copper grids (3 mm) was dipped in this SWNT-PEI 0.2-HAV and HAV dispersions, respectively, for few seconds. Then, the solvent was evaporated with the aid of an IR lamp. 

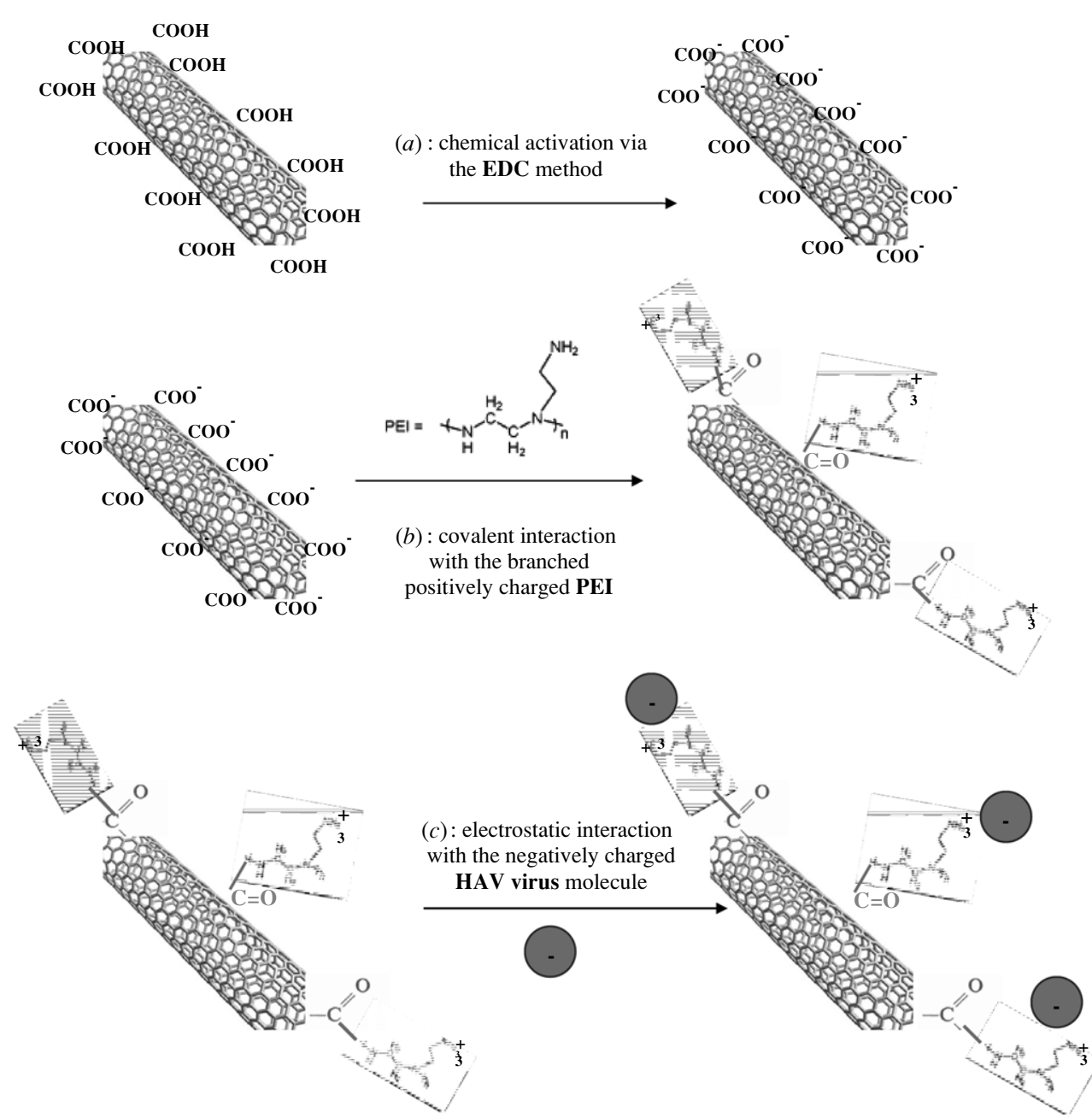

Figure 1. (a) Chemical activation of the carboxylic groups present on the SWCNT's walls, via the EDC method; $(b)$ covalent interaction between the activated SWCNT-COO ${ }^{-}$and PEI (i.e. a positively charged polymer) and (c) electrostatic interaction between PEI and the negatively charged HAV virus molecule.

\subsection{Z-potential}

Samples were analysed, after proper dilution in PBS in order to obtain the best value of scattered light intensity, by means of laser-Doppler microelectrophoresis (Zetasizer 5000, Malvern, UK). Results are reported as average of five consecutive measurements. The zeta potential was calculated by the Malvern proprietary software (version 1.36) using the following parameters: solvent viscosity $0.917 \mathrm{cP}$; dielectric constant 79.0; $\mathrm{F}(\mathrm{ka})=1.5$ (Smoluchowsky model); fifth-order fitting; laser wavelength $633 \mathrm{~nm}$; temperature $25.0{ }^{\circ} \mathrm{C}$; cell position $14.6 \%$. The microelectrophoresis cell was aligned before each measurement by using standard latex beads (from Malvern; zeta potential $-50 \pm 5 \mathrm{mV}$ ).

\subsection{Energy dispersive $x$-ray diffraction}

The x-ray experiments were carried out using an energy dispersive $\mathrm{x}$-ray [18, 19]. Incident polychromatic x-ray radiation was used and the diffracted beam is energy resolved by a solid-state detector located at a suitable scattering angle. The diffractometer operates in vertical $\theta=\theta$ geometry and is equipped with an $\mathrm{x}$-ray generator (W target), a collimating system, step motors and a solid-state detector connected via an electronic chain to a multichannel analyser. The $\mathrm{x}$-ray source is a standard Seifert tube operating at $45 \mathrm{kV}$ and $35 \mathrm{~mA}$ whose Bremsstrahlung radiation is used whereas the detecting system is composed of an EG\&G liquid-nitrogen-cooled ultrapure Ge solid-state detector connected to a PC through ADCAM hardware. Both the x-ray tube and the detector can rotate around their common centre where the sample holder is placed. After a preliminary set of measurements, the scattering angle, $\theta=3.08$, was selected to investigate the interesting range of the reciprocal space.

The sample holder is a silicon wafer where the dispersion of treated nanotubes was deposited. The solvent was allowed to dry and measurements were carried out. Checks were made to ensure that no structural modifications or damage occurred during the acquisition time.

\subsection{Reverse transcript-polymerase chain reaction test}

One hundred $\mu \mathrm{l}$ of the reaction nanotube plus virus was extracted using the Trizol LS Reagent (Invitrogen, Milan, Italy) according to the manufacturer's instructions. The final 
Table 1. The \% amount of the grafted PEI on the SWCNTs.

\begin{tabular}{lllllll}
\hline $\begin{array}{l}\text { Pure PEI } \\
\text { wt }(\%)\end{array}$ & $\begin{array}{l}\text { SWCNT-pristine } \\
\text { wt }(\%)\end{array}$ & $\begin{array}{l}\text { SWCNT-COOH } \\
\text { wt }(\%)\end{array}$ & $\begin{array}{l}\text { SWCNT/PEI 1:2.5 } \\
\text { wt }(\%)\end{array}$ & $\begin{array}{l}\text { SWCNT/PEI 1:1 } \\
\text { wt }(\%)\end{array}$ & $\begin{array}{l}\text { SWCNT/PEI 1:0.5 } \\
\text { wt (\%) }\end{array}$ & $\begin{array}{l}\text { SWCNT/PEI 1:0.2 } \\
\text { wt (\%) }\end{array}$ \\
\hline $\mathrm{d}$ & $\mathrm{n}$ & $\mathrm{n}$ & 8 & 6 & 1 & 0.75 \\
\hline
\end{tabular}

$\mathrm{d}$ : The pure PEI degraded completely; $\mathrm{n}$ : the weight loss is negligible.

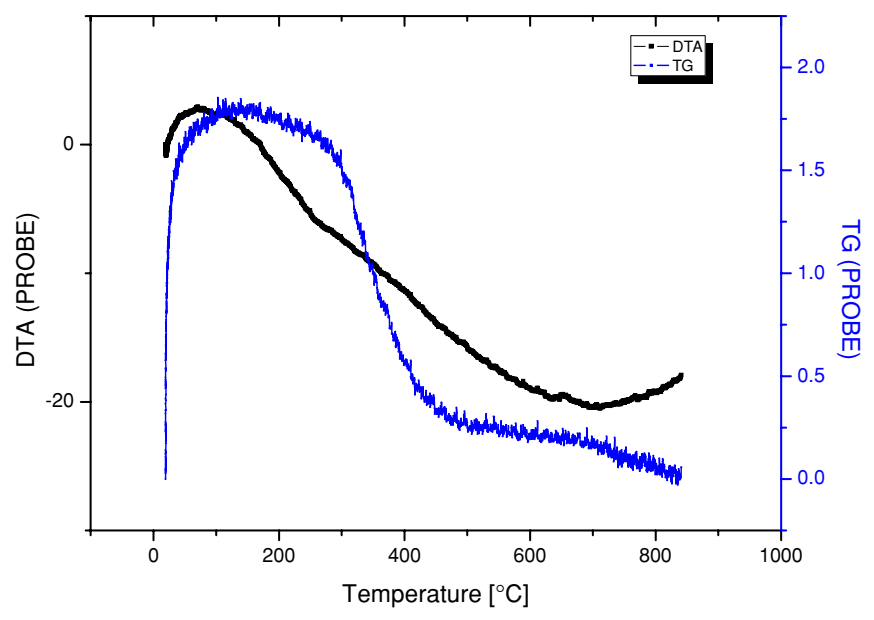

Figure 2. TGA curve (lower blue line) and DTA profile (upper black line) of SWCNT/PEI composite 1:2.5\% w/w.

viral RNA was in pellet form and was then resuspended in $10 \mu \mathrm{l}$ of RNase-DNase free water and immediately reverse transcripted (cDNA) with the specific primers. The total cDNA was amplified using specific primers designed on the VP1-VP2 region of viral genome by OligoExplorer 1.2 software. All the enzymes for the molecular tests were obtained from Promega (Milan, Italy). The conditions for reverse transcription and the amplification product have been previously described [20]. The amplified products (265 bp) were visualized by electrophoresis in $2 \%$ agarose gel (Euroclone, Milan, Italy) with ethidium bromide staining.

\section{Results and discussion}

\subsection{Differential thermal analysis and thermogravimetric analysis characterization study}

In figure 2, a representative TGA/DTA profile, performed under nitrogen, for the SWCNT/PEI composite 1:0.5\% w/w, has been reported. In particular, the relative amount of PEI grafted onto the surfaces of SWCNTs was investigated by thermogravimetric analysis (TGA) performed under nitrogen and in air. Pristine SWCNTs were thermally stable up to $600{ }^{\circ} \mathrm{C}$, whereas pure PEI degraded completely at about $500{ }^{\circ} \mathrm{C}$. At $500{ }^{\circ} \mathrm{C}$, pristine SWCNTs, COOH-SWCNTs, SWCNT/PEI composite $1: 2.5 \% \quad \mathrm{w} / \mathrm{w}, \quad$ SWCNT/PEI composite 1:1\% w/w; SWCNT/PEI composite 1:0.5\% w/w and finally, SWCNT/PEI composite 1:0.2\% w/w showed negligible, about $8 \%, 6 \%, 1 \%$ and $0.75 \%$ weight losses, respectively (see table 1 ). Thus, the amount of PEI, contained in the SWCNT/PEI composites used here was calculated as the difference between the $\%$ weight losses detectable for all the SWCNT/PEI composites (as i.e. 1:2.5; 1:1; 1:0.5 and $1: 0.2 \% \mathrm{w} / \mathrm{w}$, respectively) and the $\%$ weight losses obtained for the SWCNTs-COOH, used here as a control (that results negligible). The results, shown in table 1, completely agree with the data described in literature [17]. Furthermore, in this study, we could quantify, for the first time, the percentage amount of grafted PEI for several \% w/w of SWCNTs and PEI in the resulting composites.

Grafting with PEI made SWCNT/PEI composites easy to disperse in water and phosphate buffer saline solution, with resulting suspension still stable after 1 year, at variance with $\mathrm{COOH}-\mathrm{SWCNT}$ which disperse poorly in water and in the phosphate buffer saline solution and precipitate within a few hours.

\subsection{Morphological characterization of SWCNTs and the modified SWCNTs}

The morphological characterization of HAV-conjugated SWCNTs/PEI (C-HAV) was performed by scanning electron microscopy. This was achieved in three steps which include the characterization of the functionalized SWCNTs$\mathrm{COOH}$ (figures $3(a),(b)$ ), the composite SWCNTs/PEI(0.5) (figures $3(c),(d)$ ) and finally the composite SWCNTs/PEI(0.2) (figures 3(e), $(f)$ ).

Figures 3(a), $(b)$ show the typical forest of SWCNTs distribution, which is also preserved when the nanotubes are functionalized with carboxylic groups. The subsequent coating with high doses of PEI polymer (i.e. 0.5) results in a complete coverage of the nanotubes as clearly shown in figures 3(c) and (d) and also widely described in our previous work [17]. The 0.2PEI/SWCNT composite material (figures $3(e)$ and $(f)$ ) shows a partial coverage of the SWCNTs because of the lower amount of the polyeletrolyte.

This composite material also has the property of homogenously dispersing in water, where it has been observed to be stable for over 6 months at room temperature, without any solid phase aggregation. This probably relates to the stability of the covalent bond between the PEI polycation and the SWCNTs-COOH, which occurs through an amidic bond (see figures $1(a)$ and $(b)$ ). Figure 4 shows a flask with a composite material dispersion after 6 months.

The morphological characterization of the conjugated system is based on the comparison of TE micrographs of the virus alone and upon conjugation with the composite material. In figure 5 the typical spherical shapes of the HAV can be identified. Their diameter was measured and found in the $20 \mathrm{~nm}$ range for a single virus particle [21].

In figure 6 the nanometric HAV viral spheres appear to be entrapped in the C-HAV network complex (as indicated by the continuous arrows). A careful inspection of the micrographs 


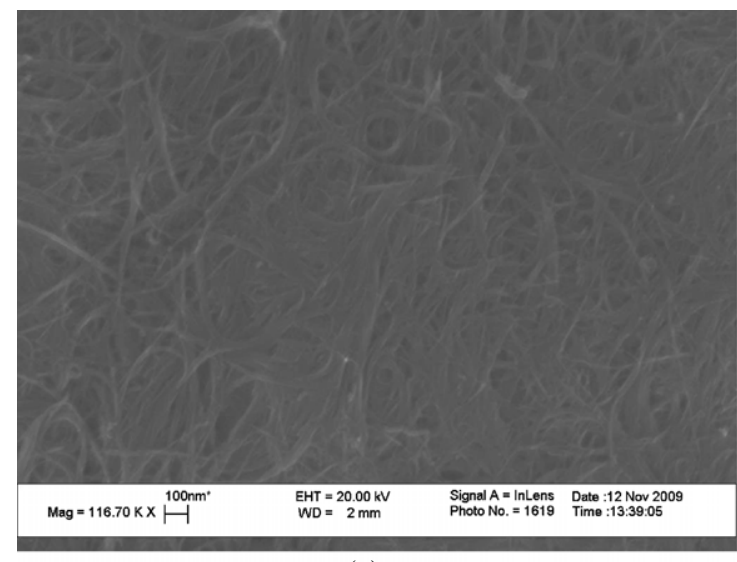

(a)

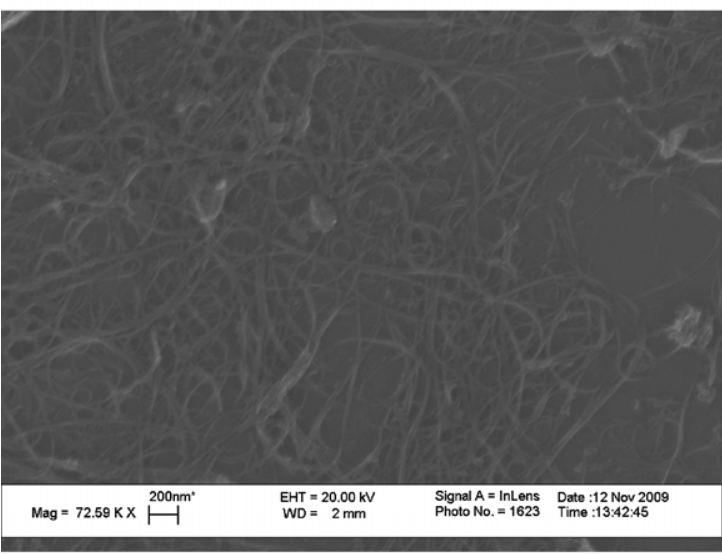

(b)

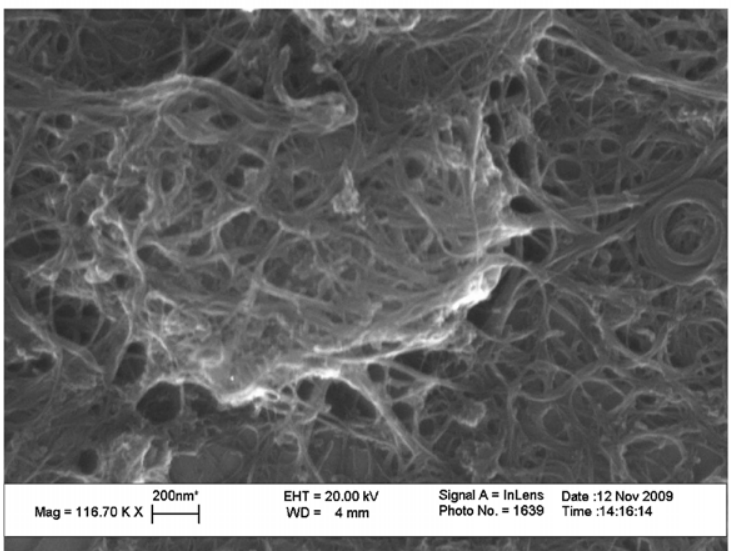

(c)
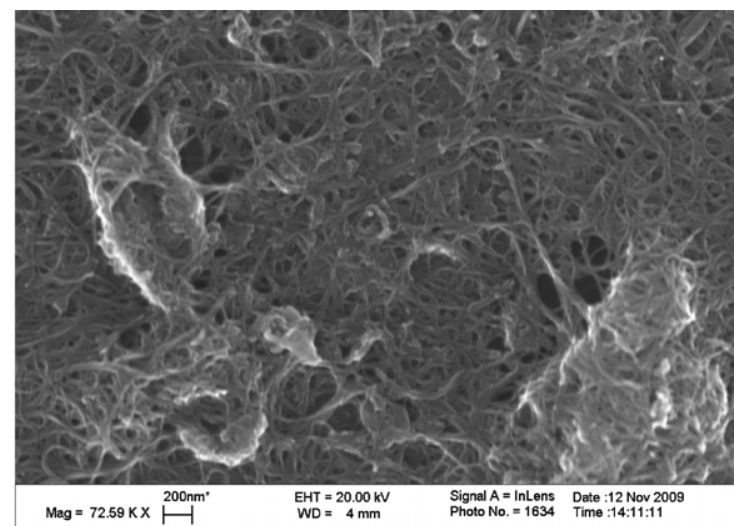

(d)

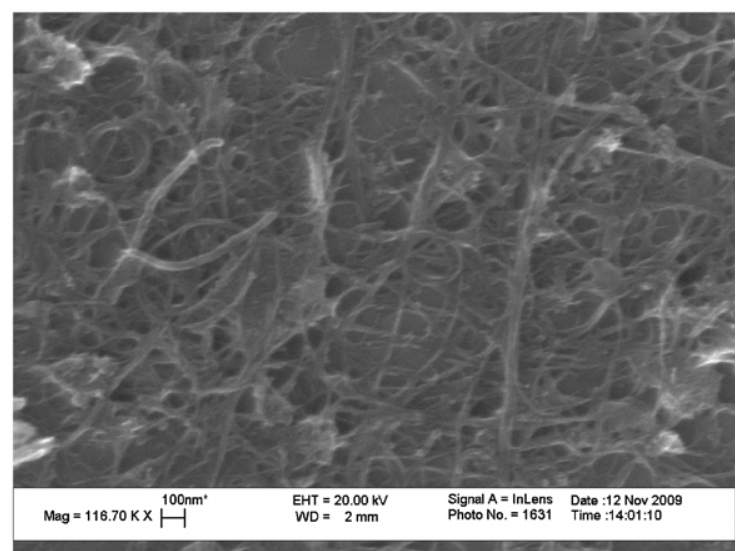

(e)

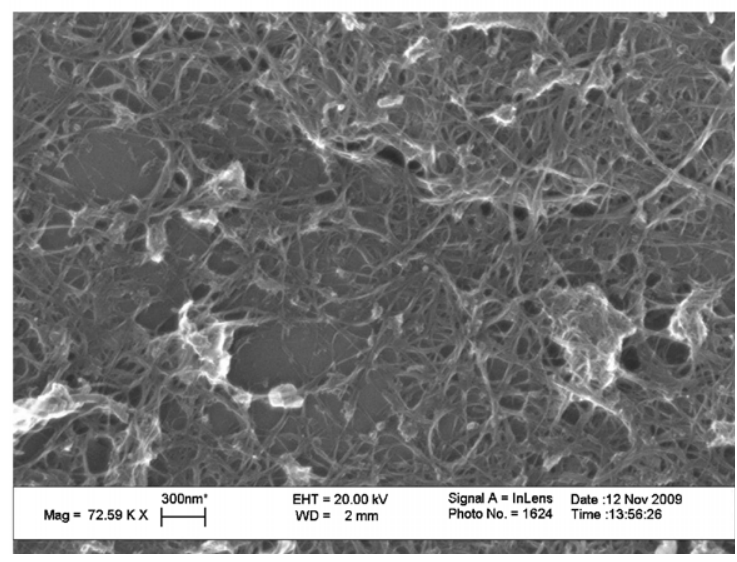

$(f)$

Figure 3. SEM micrographs of (a) SWCNTs-COOH (magnification of $116.70 \mathrm{KX}$ ); (b) SWCNTs-COOH (magnification of $72.59 \mathrm{KX}$ ); (c) 0.5PEI-coated SWCNT (magnification of $116.70 \mathrm{KX}) ;(d)$ 0.5PEI-coated SWCNT (magnification of 72.59 KX); $(e)$ 0.2PEI-coated SWCNT (magnification of $116.70 \mathrm{KX}$ ) and $(f)$ 0.2PEI-coated SWCNT (magnification of $72.59 \mathrm{KX}$ ).

shows that the PEI structure entangled around the nanotubes has a peculiar inner spherical structure (as indicated by the dotted arrows), with a diameter of $20 \mathrm{~nm}$, compatible with the interaction with the hepatitis A virus.

The possibility of detecting conjugated particles at small scale was first exploited by Cricenti et al [22] who observed the interaction between lymphocyte cells and the human immunodeficiency virus - HIV-by atomic force microscopy (AFM) in the repulsive regime of the contact mode. After exposure of the lymphocyte to HIV a decrease in surface protrusions (lost of microvilli) and the creation of many dips was observed. Some particles, 120-130 nm size, were also observed in proximity of the cell surface and these values are consistent with the expected dimensions of HIV, thus suggesting the viral origin of these spherical particles.

Some larger structures ascribable to virus-nantotube interactions may be present as well. In a recent paper Yu et al [16] took SEM micrographs of DNA conjugated with mostly short MWCNTs coated with PEI-MWCNTs (a solution of PEI-MWCNTs $50 \mu \mathrm{g} \mathrm{mL}^{-1}$ in phosphate buffer solutions 


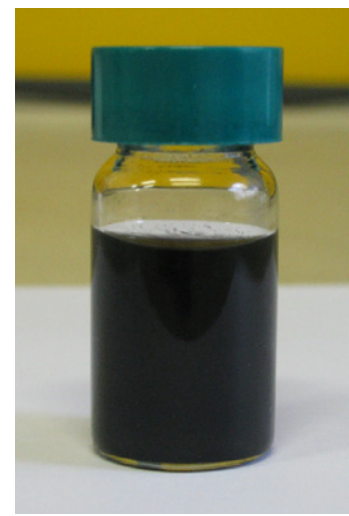

Figure 4. Aqueous dispersion of $1 \mathrm{mg} \mathrm{mL}^{-1} \mathrm{SWCNT} / \mathrm{PEI}$ composite, at room temperature.

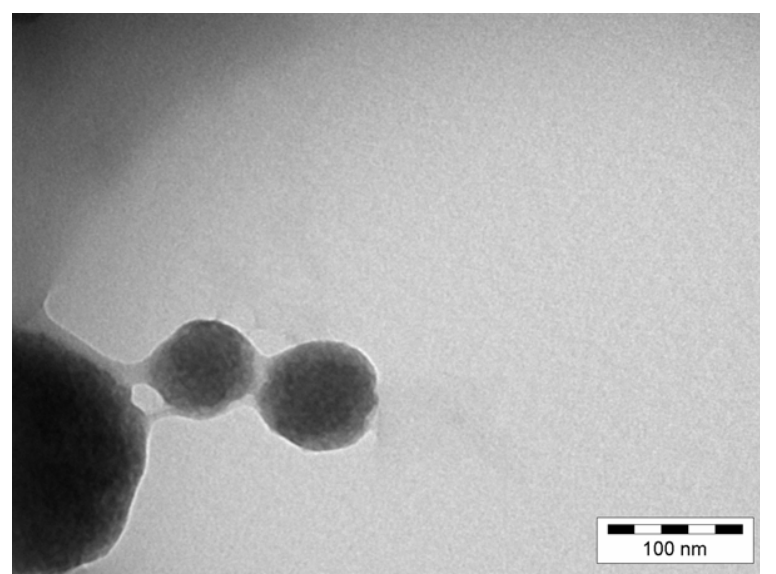

Figure 5. TEM micrographs of clusters of the HAV. The smallest cluster shows a diameter of $40 \mathrm{~nm}$ (where a single HAV particle has a diameter of $\sim 20 \mathrm{~nm}$ ), while the largest cluster have a diameter of $200 \mathrm{~nm}$.

(PBS)) which show irregular spherical structures between 50 and $150 \mathrm{~nm}$ in diameters, typically obtained due to degrees of plasmid condensation depending on the charge density, the hydrophobic character of the interaction and the number of pDNA molecules in the condensate [16].

In our case, we observe round areas of diameters ranging from $\sim 40$ to $170 \mathrm{~nm}$, ascribable to the interacting virus particles. However, care must be taken in the attribution, because of the excess of PEI used for coating the nanotubes, which might give a contribution to the larger structures.

Similarly, in this case we observe the presence of round particles in proximity of the PEI coated nanotubes suggesting their viral origin.

More studies were performed on the C-HAV system, i.e. measurements of $Z$-potentials and this investigation adds information in the same direction.

The values of the Z-potential of the SWCNT-COOH sample, the SWCNT/PEI complex and the C-HAV conjugated sample are reported in table 2 . The first sample displays a negative value of the surface charge [17]. The coating with the polycationic PEI results into a reverting of the $Z$-potential to a positive value, which is further modified towards less positive values upon exposure of the composite material to the HAV.

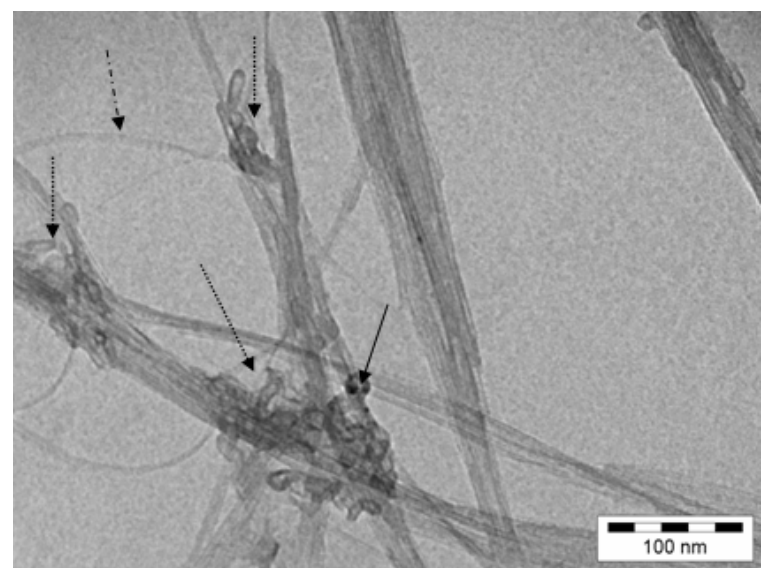

Figure 6. TEM micrographs. The continuous arrow indicates the HAV spherical particles; the dotted arrows show the PEI coating on the carbon nanotubes and finally, the dotted and continuous arrows indicate the single wall carbon nanotube bundles.

Table 2. Z-potential of the carboxylic functionalized SWCNTs (i.e.; SWCNT-COOH), upon coating with PEI (i.e. SWCNT/PEI) in excess and subsequent conjugation with the HAV virus (i.e. C-HAV).

\begin{tabular}{ll}
\hline Samples & Z-potential \\
\hline SWCNT-COOH & $-42.2 \pm 0.5 \mathrm{mV}$ \\
SWCNT/PEI & $+18.6 \pm 0.2 \mathrm{mV}$ \\
C-HAV & $+16.5 \pm 0.3 \mathrm{mV}$ \\
\hline
\end{tabular}

This is in line with an overall process where the nanotubes are first functionalized with carboxylated groups, which imprint a negative charge on the surface. Then, following the covering of the nanotubes with an excess of polycationic PEI, the surface charge reverts because of the presence of the ammonium heads of the branches. The now positively charged surface of the composite material may interact with the HAV, which bears a negative charge, by electrostatic interactions. We, consequently, observe a decrease of the total positive surface charge, clearly indicating that the conjugation of the composite material and PEI occurred. The net balance of charges is still positive, due to the large excess of PEI used in the coating that dominates the total charge.

\subsection{EDXD characterization of SWCNTs conjugated with virus}

Obtaining PEI-coated SWCNTs conjugated with the HAV follows two steps, both monitored through EDXD: the coating with PEI and the subsequent conjugation. Considering the branched nature of the polymer used here, which may create a thick network between nanotubes (see figure $3(b)$ ), effectively hindering of further conjugation, in this paper we opted for SWCTNs:PEI wt ratios of 1:0.2 and 1:0.5. Furthermore, it was carefully checked that no residual material from the virus growth procedure would make a significant contribution in the same $q$ range and affect the quality and interpretation of the data. 


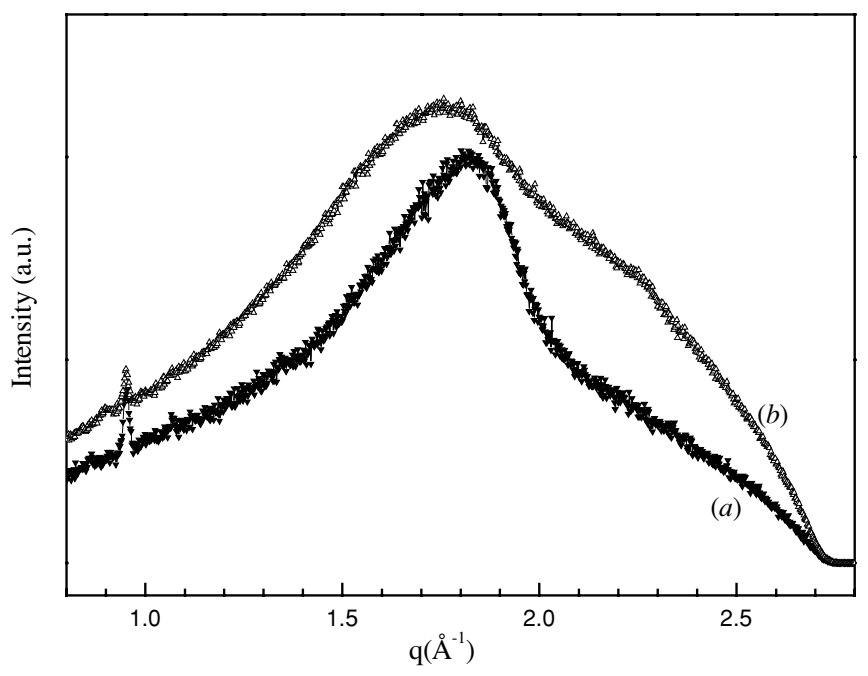

Figure 7. XRD patterns of $(a)-\mathrm{COOH}$ functionalized SWCNTs and $(b)$ 0.2PEI-coated SWCNTs.

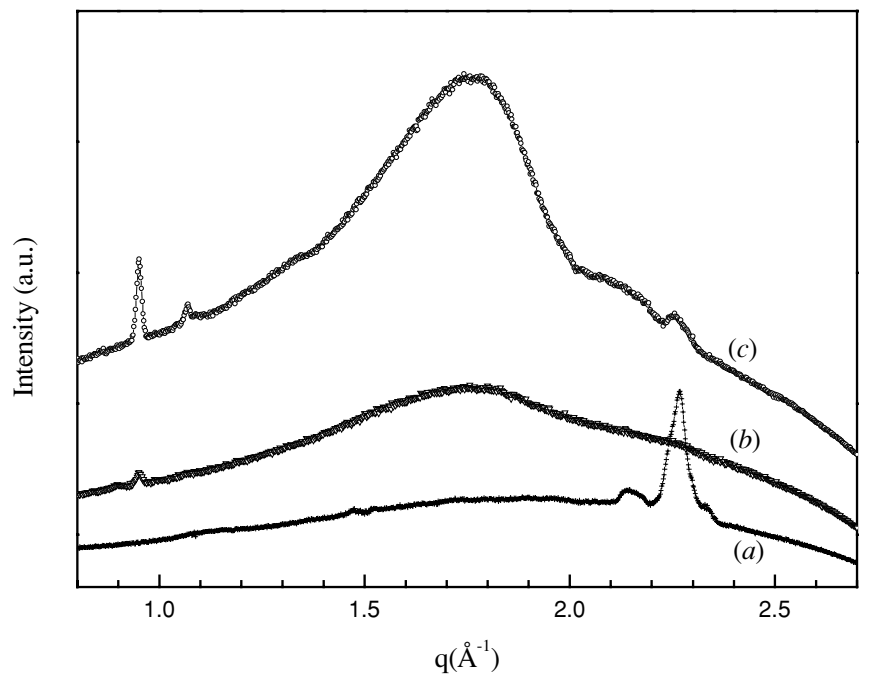

Figure 8. XRD patterns of $(a)$ HA virus, $(b)$ 0.2PEI-coated SWCNTs, $(c)$ 0.2PEI-coated SWCNTs conjugated with HAV.

After exploring a few ranges of the reciprocal space, we eventually opted for the region $0.8-2.7 \AA^{-1}$ because it appeared that all single contributions of the separate materials, i.e. nanotubes, polymer and virus, could easily be singled out.

In all figures, the spectra are arbitrarily vertically shifted, to render them clearer. Furthermore, the sharp peaks, appearing in some of the spectra on the left-hand side, are fluorescence peaks related to the residual $\mathrm{Fe}$ and Co metals, used in the nanotube preparation. Their amount was random in the samples. In figure 8 the diffraction pattern of the $-\mathrm{COOH}$ functionalized SWCNTs is plotted together with the analogue coated with $0.2 \mathrm{wt} \%$ PEI. The main peak of the functionalized nanotubes occurs at about $1.81 \AA^{-1}$ and corresponds to the $\mathrm{C}-\mathrm{C}$ interaction between $\mathrm{C} 1$ and $\mathrm{C} 4$ in the exagonal rings constituting the nanotubes [23]. Coating with the PEI has three effects on the overall spectrum: the apparent shift of the peak from 1.81 to $1.76 \AA^{-1}$, the broadening of the peak and the appearance of a shoulder at

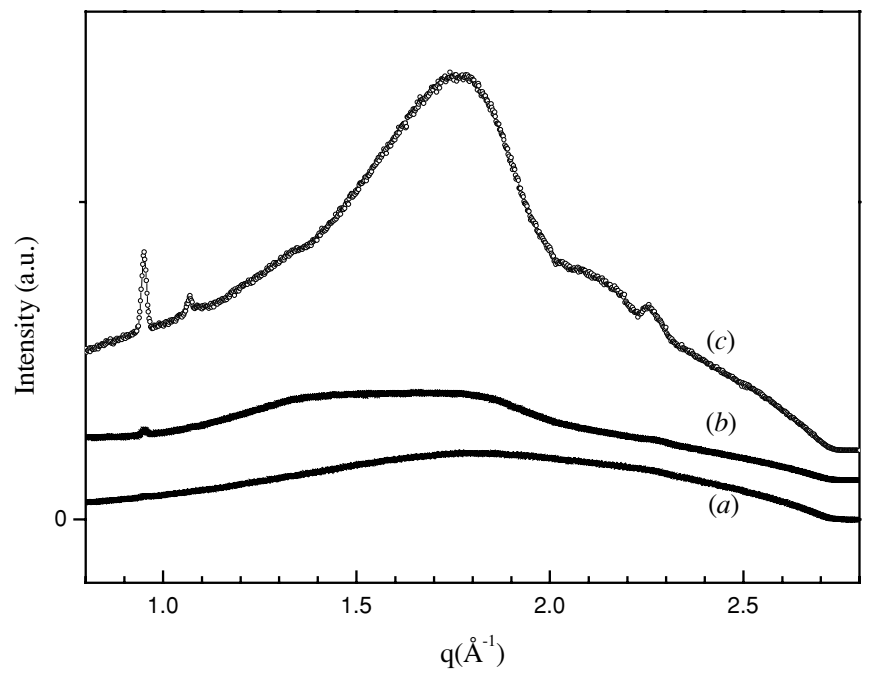

Figure 9. XRD patterns of $(a)$ growth medium of the HAV conjugated with SWCNTs, $(b)$ bovin serum with SWCNTs and (c) 0.2PEI-coated SWCNTs conjugated with HAV.

$2.25 \AA^{-1}$. These are all observations compatible with covering the nanotubes with a polymer with a wider distribution of distances in this region. The flexibility of the polymer chain and its adaptation to the nanotube surface provide a large set of interactions corresponding to distances around $3.5 \AA$, with the overall effect of broadening the peak. The shoulder at $2.25 \AA^{-1}$ is, instead, typical of the $\mathrm{C} \alpha$ and $\mathrm{N}$ interaction in the immidic bond.

Figure 7 shows the $x$-ray diffraction pattern of the 0.2PEIcoated SWCNTs conjugated with the HA virus, in comparison to the patterns of both the $0.2 \mathrm{PEI}$-coated material and the virus alone.

In figure $8(a)$ the spectrum of the virus alone shows two peaks at about 2.14 and about $2.27 \AA^{-1}$, which can be ascribed to contact distances between non-consecutive atoms within the protein structure of the virus. Since such a structure is more ordered than that of the flexible chain of the PEI, the distance distribution is narrower and the virus peaks are clearly distinguishable.

In figure $8(c)$ it can be clearly observed that the virusrelated peaks also persist in the spectrum of the conjugated material, though at a lower intensity, providing a clear indication that the conjugation process is effective and that the virus, indeed, remains in contact with the coating PEI upon conjugation. However, care must be taken that what we observe is not an artefact related to residuals of the sample preparation. In particular, an insufficient purification of the viruses, following the growing of the colonies, may result in spurious peaks in the diffraction patterns. Therefore, we went through the virus-growing procedure and performed some test measurements with 0.2PEI-coated SWCNTS conjugated with the virus-growing material, i.e. the growth medium and the bovine serum. The outcome is shown in figure 9, together with the reference 0.2PEI-coated SWCNTS-HAV sample. Such materials do not provide any sharp structures in the region of interest around $2.2 \AA^{-1}$. Therefore, even if present in our sample, in spite of the purification procedure, they would not interfere with the XRD patterns. 


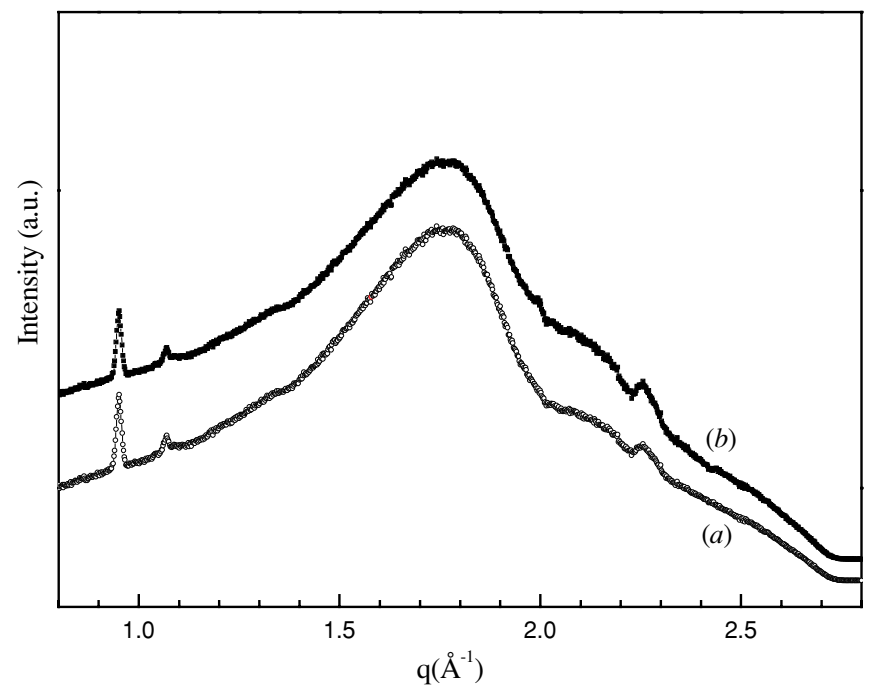

Figure 10. XRD patterns of (a) 0.2PEI-coated SWCNTs conjugated with HAV and $(b)$ 0.5PEI-coated SWCNTs conjugated with HAV.

Finally, we checked the effect of a higher dose of PEI, i.e. $0.5 \%$ wt to the overall virus uptake, though keeping the dose of conjugating virus constant. In figure 10, the diffraction pattern reported the 0.2- and 0.5PEI-coated samples, conjugated with the HAV. The broadness of the peaks does not allow a quantitative assessment. However, the peaks at 2.14 and about $2.27 \AA^{-1}$ indicative of the virus presence are more intense with respect to the main nanotube peak, for the 0.5PEI sample, hinting at a higher virus uptake in correspondence of a higher PEI dose. The reference 0.5PEI-SWCNTs pattern was also collected (though not shown in the figure for clarity sake) and shows a small increase in the areas around $2.25 \AA^{-1}$.

\subsection{RT-PCR investigation of the SWCNTs conjugated with the HAV virus}

The RT-PCR was used for 1:2.5, 0.2 and 0.5PEI coated conjugated to the HAV as control over the diffraction experiments.

The presence of HAV attached to SWCNTs functionalized with PEI has been demonstrated by the presence of the virus specific amplification band (figures $11(a)$ and $(b)$ ). Depending on the primers used the amplified product was a $265 \mathrm{bp}$ (base pair) band. To be sure about the amplification band, the control virus was used in parallel giving the correct position of the band. The negative control, included in each test, confirms the absence of cross-contamination among the samples giving false positive results. The two amplification bands using SWCNT-PEI ratios 1:2.5, 1:0.5 and 1:0.2 appear to be of the same intensity but no attempt was made to quantify the band intensity.

The molecular method, RT-PCR, after amplification, confirms the presence of HAV on the nanotubes, stable over 1 h. The use of Trizol LS reagent, containing phenol, causes the complete destruction of virus including the eventually virus particles inside the SWCNTs. The specificity of the reaction about the presence of the virus is confirmed both by the used primers and by the band position as demonstrated by the control virus.

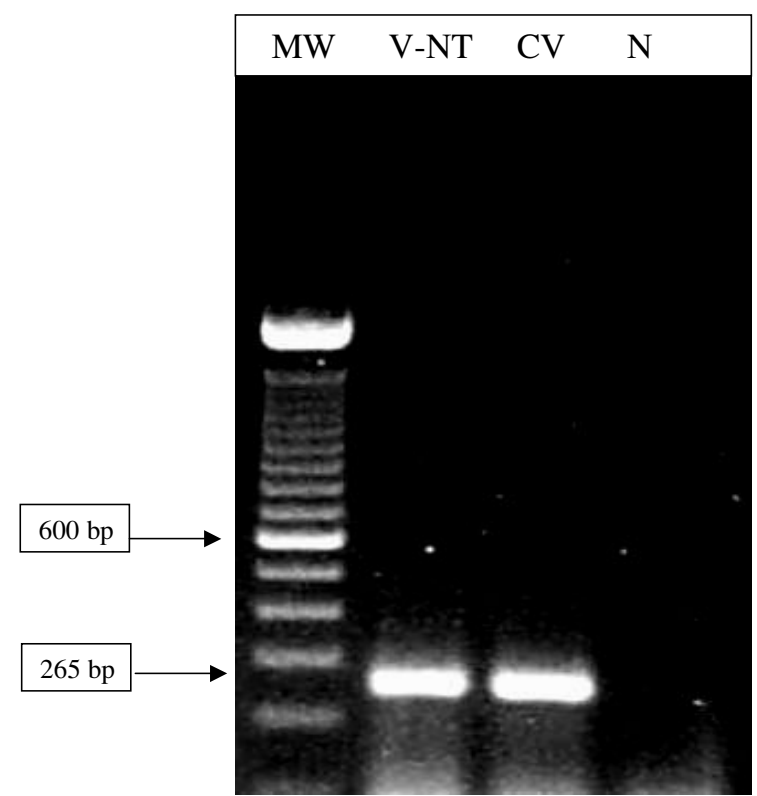

(a)

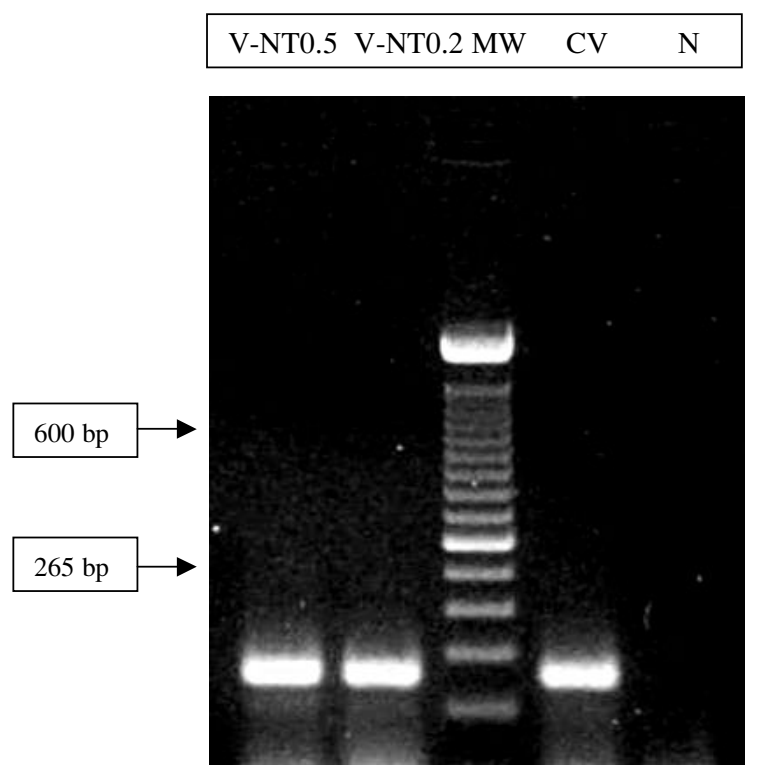

(b)

Figure 11. (a) MW (molecular weight): Invitrogen, 100 base pairs (base pair) DNA Ladder $1.0 \mu \mathrm{g} \mu \mathrm{l}^{-1}$; V-NT: $1 \times 10^{5}$ particles/test of hepatitis A virus HM175 + SWCNT/PEI 2.5; CV: positive control virus (laboratory strain HAV); N: negative control (PCR reaction performed with water). (b) V-NT 0.5 (Virus-SWCNT-PEI): $1 \times 10^{5}$ particles/test of hepatitis A virus HM175 + SWCNT-PEI 1:0.5; V-NT 0.2 (Virus-SWCNT-PEI): $1 \times 10^{5}$ particles/test of hepatitis A virus HM175 + SWCNT-PEI 1:0.2; MW (Molecular weight): Invitrogen, $100 \mathrm{bp}$ (base pair) DNA Ladder $1.0 \mu \mathrm{g} \mu \mathrm{l}^{-1}$; CV: positive control virus (laboratory strain HAV 175); N: negative control.

\section{Conclusions}

The surface chemistry of SWCNTs offers the possibility of introducing more than one function on the same tube and also to wrap the walls of these carbon cylinders with biocompatible polymers so that targeting molecules, contrast agents, drugs, or viruses can be used at the same time. This has opened up new 
horizons in the study of the biological properties of SWCNTs. In this paper, we focused on the possibility of conjugating the SWCNT/PEI high load-composite with the HAV virus, which can be useful to perform transfection experiments in vitro. For the first time, to our knowledge, in this paper we have shown through SEM, Z-potential, EDXD measurements and RT-PCR that the conjugation between SWCNT/PEI and HAV occurs.

In particular, the comparative EDXD investigation demonstrates that the nanotubes coated with low doses of PEI (i.e. 0.2 and $0.5 \mathrm{wt} \%$ with respect to the nanotubes) successfully conjugate with the virus. All different samples, in fact, show distinctive peak shapes, that allow clearcut assignment. We can exclude possible interferences in the measurements related to the virus growth procedure. Furthermore, a higher virus uptake can be hypothesized when the 0.5 PEI sample is used. RT-PCR measurements confirmed that the conjugation of the coated nanotubes and HAV occurred and is stable for over $1 \mathrm{~h}$.

The final goal is the use of the nanotubes as a carrier in transfection processes, since HAV cannot multiply in cell lines where the specific receptors are absent on the cellular membrane.

\section{References}

[1] Itkis M E, Yu A and Haddon R C 2008 Nano Lett. 82224

[2] Milne W I, Teo K B K, Amaratunga G A J, Legagneux P, Gangloff L, Schnell J P, Semet V, Thien Binh V and Groening O 2004 J. Mater. Chem. 14933

[3] Banerjee S and Stanislaus S W 2002 J. Am. Chem. Soc. 1248940

[4] Porter D, Sriram K, Wolfarth M, Jefferson A, Schwegler-Berry D, Andrew M E and Castranova V 2008 Nanotoxicology 2144

[5] Martin C R and Kohli P 2003 Nat. Rev. Drug Discov. 229
[6] Bianco A and Prato M 2003 Adv. Mater. 151765

[7] Shi K N W and Dai H 2005 J. Am. Chem. Soc. 1276021

[8] Prato M, Kostarelos K and Bianco A 2008 Acc. Chem. Res. 4160

[9] Pantarotto D, Hoebeke J, Graff R, Partidos C D, Briand J-P, Prato M and Bianco A 2003 J. Am. Chem. Soc. 1256160

[10] Pantarotto D, Partidos C D, Hoebeke J, Brown F, Kramer E, Briand J-P, Muller S, Prato M and Bianco A 2003 Chem. Biol. 10961

[11] Novina C D, Murray M F, Dykxhoorn D M, Beresford P J, Riess J, Lee S K, Collman R G, Lieberman J, Shankar P and Sharp P A 2002 Nat. Med. 8681

[12] Klumpp C, Kostarelos K, Prato M and Bianco A 2006 Biochim. Biophys. Acta 1758404

[13] Hu H, Ni Y, Mandal S K, Montana V, Zhao B, Haddon R C and Parpura V 2005 J. Phys. Chem. B 1094285

[14] Liu Y, Wu D-C, Zhang W-D, Jiang X, He C-B, Chung T S, Goh S H and Leong K W 2005 Angew. Chem. Int. Ed. 444782

[15] Boussif O, Lezoualch F, Zanta M A, Mergny M D, Scherman D, Demeneix B and Behr J P 1995 Proc. Natl Acad. Sci. USA 927297

Robaczewska M et al 2001 Gene Ther. 8874

[16] Yu B-Z, Ma J-F and Li W-X 2009 Nat. Proc. hdl:10101/npre.2009.2753.1 (posted 5 Jan)

[17] Valentini F, Amadei M, Palleschi G and Carbone M 2009 Int. J. Environ. Health 3 285-301

Liu Y, Wu D-C, Zhang W-D, Jiang X, He C-B, Chung T S, Goh S H and Leong K W 2005 Angew. Chem. Int. Ed. 44 4782-5

[18] Matassa R M, Carbone, Laceri R, Purrello R and Caminiti R 2007 Adv. Mater. 193961

[19] Caminiti R, Ballirano P and Carbone M 2006 J. Macromol. Sci. B 451005

[20] Macaluso A, Petrinca A, Lanni L, Saccares S, Amiti S, Gabrieli R and Dovizia M 2006 J. Food Prot. 69449

[21] Wheeler C M, Robertson B H, Van Nest G, Dina D, Bradley D W and Fields H A 1986 J. Virol. 58307

[22] Cricenti A, Generosi R, Girasole M, Scarselli M A, Bach S, Colizzi V and Perfetti P 1999 J. Vac. Sci. Technol. A 171141

[23] Rinzler A G et al 1998 Appl. Phys. A 6729 